\title{
1 Introduction to Evolutionary Theory and Its Modern Application to Human Behavior and Cognition
}

\author{
Aaron T. Goetz and Todd K. Shackelford
}

Darwin (1859) was not the first to suggest that species evolve. In fact, one of the first discussions of evolution predates Darwin by two and a half millennia. Anaximander, a Greek philosopher, suggested that "in water the first animal arose covered with spiny skin, and with the lapse of time some crawled onto dry land and breaking off their skins in a short time they survived." What Darwin (1859) provided, however, was a viable working mechanism of evolution: natural selection. Darwinian selection has become the centerpiece of biology, and in the last few decades, many psychologists and anthropologists have recognized the value of employing an evolutionary perspective in their work (e.g., Barkow, Cosmides, \& Tooby, 1992; Chagnon \& Irons, 1979; Daly \& Wilson, 1983; Symons, 1979). With a focus on evolved psychological mechanisms and their information processing, evolutionary psychology has risen as a compelling and fruitful approach to psychological science. This chapter provides an introduction to evolution by natural selection and its modern application to the study of human behavior and cognition.

\section{Natural Selection and Sexual Selection}

Evolution by natural selection is the process that results when (1) individuals of a population vary in their characteristics, (2) much of the variation is heritable, and (3) resources are limited, so that individuals reproduce differentially (Darwin, 1859; Mayr, 1982). Individuals can vary morphologically, physiologically, psychologically, and behaviorally-no two individuals are exactly the same. Because of these variations, some individuals may be better able to survive and reproduce in their current environment than other individuals. If the variations are heritable (i.e., if they have a genetic component), the characteristics can 
be passed down from parents to offspring. Limited resources (e.g., food, available mates) result in a competition between individuals, and those individuals who have inherited characteristics that allow them to compete more effectively will produce more offspring. Thus, all organisms are subject to evolution by natural selection. As long as the ingredients of natural selection are present-variation, heredity, and competition resulting in differential reproduction-organisms will evolve. An example of natural selection follows.

The peppered moth (Biston betularia) is typically white with black spots. This coloration provides an effective camouflage for the moths as they rest on certain birch trees. There exists variation in the coloration of moths so that some are very white and some very black. In a series of studies, Kettlewell $(1955,1956)$ documented that when the white trees on which the moths rested became dark from industrial pollution, birds ate more of the white moths because they were now conspicuous on the soot-covered trees. In polluted areas, the population of darker, or melanic, moths replaced the lighter form, but in unpolluted areas, more of the light-colored moths survived. Kettlewell showed that the environment in which the moths were better camouflaged contributed to better survival and reproduction. Kettlewell's work is a classic demonstration of natural selection in action.

Herbert Spencer's summary of natural selection, "survival of the fittest," has, unfortunately, caused more confusion than clarification (Gaulin \& McBurney, 2004). Reproduction is a much larger component of natural selection than is survival. If an individual had characteristics that enabled it to survive for hundreds of years yet it never reproduced, those characteristics could not be favored by selection because without transmission to offspring, characteristics cannot become more common in a population. Survival, therefore, functions only to enable individuals to reproduce (directly or indirectly). Second, Spencer's adage suggests that an individual may evolve to be the "fittest." What determines whether an individual is fit is its design in relation to competing designs in the current environment. What is fit in one generation may be unfit in another generation. Also, fit is often taken to imply physically fit. Fitness, in an evolutionary context, is an organism's success in producing offspring that survive to reproductive age (Williams, 1966).

Sexual selection is the process that favors an increase in the frequency of alleles associated with reproduction (Darwin, 1871). Darwin distinguished sexual selection from natural selection, but today most evolutionary scientists combine the two concepts under the label natural 
selection. Sexual selection is composed of intrasexual competition (competition between members of the same sex for sexual access to members of the opposite sex) and intersexual selection (differential mate choice of members of the opposite sex). Under sexual selection, even a trait that is a liability to survival can evolve. When the sexual attractiveness, for example, of a trait outweighs the survival costs to maintain it, the trait may be sexually selected. The epitome of a sexually selected trait is the peacock's tail. Maintaining and maneuvering an unwieldy tail is metabolically costly for peacocks, and it is often the target of predators. The cumbersome tail evolved, however, because it was attractive to peahens. The mass and brightness of the plumage are attractive to peahens because this appearance signals a modicum of parasites (Hamilton \& Zuk, 1982). Peacocks with smaller, lackluster tails are more susceptible to parasites and have a higher parasite load. Thus, the large, bright tail feathers are an honest signal of health, and peahens would be reproductively wise to select as mates males with such tails (who sire offspring that share their high-quality genes).

In many species, particularly polygynous species where male reproductive variance is high and female reproductive variance is low, sexual selection is responsible for prominent sexual dimorphism. In such species, intrasexual competition between males for sexual access to females is fierce, and a size advantage is adaptive. It is often difficult to establish whether a trait evolved via natural selection or sexual selection, but, as mentioned previously, this distinction is not often necessary.

In summary, the core premise of natural selection as a mechanism for evolution is that individual variation exists among traits in a population as a result of random mutations. Those individuals who have traits that better enable them to survive and reproduce will propagate the genes associated with those traits throughout the population.

\section{The Modern Synthesis and Inclusive Fitness Theory}

The details of modern evolutionary theory, or neo-Darwinian theory, are the result of the modern synthesis. From the early 1930s to the 1950s, advances in genetics, systematics, and paleontology aligned Darwin's theory with the facts of genetics (Mayr \& Provine, 1980). The modern synthesis is so called because it was the integration or synthesizing of Darwinian selection with Mendelian genetics. R. A. Fisher, J. B. S. Haldane, Sewall Wright, Ernst Mayr, and Theodosius Dobzhansky are considered the primary authors of the modern synthesis (Mayr \& 
Provine, 1980). With a more precise understanding of inheritance, Darwin's theory of evolution by natural selection took flight as a powerful explanatory model.

Following the modern synthesis, evolution by natural selection was extended once more to include inclusive fitness theory (Hamilton, 1964). Hamilton reasoned that selection could operate through classic fitness (i.e., the sum of an individual's own reproductive success) and inclusive fitness, which includes the effects of an individual's actions on the reproductive success of genetic relatives. That is, a trait will be naturally selected if it causes an individual's genes to be passed on, regardless of whether the individual directly produces offspring. This addendum to natural selection produced a "gene's eye" view of selection and could now explain the evolution of altruistic behavior (i.e., behavior that is beneficial to others but costly for the actor). Genes associated with producing an alarm call when sighting a predator, for example, may spread throughout a population even when is detrimental to the caller if the alarm call is emitted in the presence of genetic relatives and has an overall benefit to those relatives (e.g., Sherman, 1977). Hamilton's inclusive fitness theory is considered the most important advance in our understanding of natural selection, so much so that the term inclusive fitness theory is synonymous with the term evolution by natural selection.

\section{The Products of Evolution: Adaptations, Byproducts, and Noise}

Although natural selection is not the only mechanism of evolutionothers include mutation, migration, gene flow, genetic drift-it is the primary means of modification and the only creative evolutionary force capable of producing functional organization (Fisher, 1954; Mayr, 1963; Williams, 1966). The creative force of natural selection, acting on random genetic variation, designs three products: adaptations, byproducts of adaptations, and noise.

Adaptations are central to the study of evolution. Through the process of natural selection, small, incremental phenotypic changes that enhance an organism's ability to survive and reproduce (relative to competing designs) accumulate to form an adaptation. Adaptations are inherited, they develop reliably, they are usually species-typical, and they were selected for because they were economic, efficient, and reliable solutions to adaptive problems (Buss, Haselton, Shackelford, Bleske, \& Wakefield, 1998; Thornhill, 1997; Tooby \& Cosmides, 1990; Williams, 1966). An 
adaptive problem is an obstacle or impediment that was recurrent during a species's evolutionary history and whose solution affected the survival and reproduction (i.e., genetic propagation) of an organism. Furthermore, adaptive problems are not necessarily "problems," they are the "regularities of the physical, chemical, developmental, ecological, demographic, social, and informational environments encountered by ancestral populations during the course of a species's or population's evolution" (Tooby \& Cosmides, 1992, p. 62). In sum, natural selection designs adaptations that solve adaptive problems associated with survival and reproduction. The function of the heart, the production of sweat, and sexual arousal are all adaptations designed by natural selection. The heart is an anatomical adaptation designed to circulate blood throughout an organism's body. The production of sweat is a physiological adaptation designed to thermoregulate an organism. Sexual arousal is a psychological adaptation designed to motivate sexual behavior.

Not all products of natural selection are adaptations. Byproducts of adaptations are characteristics of a phenotype that are functionless and do not solve adaptive problems. They are called byproducts because they are incidentally tied to adaptations and are therefore "carried along" with them. Identifying byproducts is as rigorous a process as identifying adaptations because the allegation that a trait is a byproduct requires the alleger to state the adaptations of which it is a byproduct. The human navel and the whiteness of bone are byproducts of adaptations-they do not contribute in any way to an individual's survival or reproduction. In keeping with our mandate: the human navel is a byproduct of an umbilical cord and the whiteness of bone is a byproduct of the calcium in bones.

The third product of evolution is noise, or random effects. Noise is also functionless and cannot solve adaptive problems. Noise can be produced by random changes or perturbations in the genetic or developmental environment or by chance mutations. Noise, unlike a byproduct, is not linked to the adaptive aspect of a characteristic. The random shape of an individual's navel is an example of noise.

In summary, the evolutionary process produces three products: adaptations, byproducts, and noise. Adaptations are the product of natural selection and are functionally organized features that contribute to a species's reproductive success, however indirectly. Byproducts and noise do not solve adaptive problems and are not subject to natural selection themselves. In the following section, we discuss how the study of 
psychological adaptations has changed the study of human behavior and cognition.

\section{Evolutionary Psychology}

Evolutionary psychology attempts to make sense of current human thought, emotion, and behavior by careful consideration of human evolutionary history. Over the course of our evolutionary history, humans have faced many adaptive problems that needed to be solved to survive and reproduce. Generation after generation, over millions of years, natural selection slowly shaped the human brain, favoring circuitry that was good at solving these adaptive problems of our ancestors. The study of psychological adaptations (or evolved psychological mechanisms) is central to evolutionary psychology.

Because the focus of evolutionary psychology is on describing adaptations, some have charged its practitioners as being hyperadaptationists. Assuming a priori that a trait may be an adaptation is an experimental heuristic that guides research questions and methodology. Biologists have been conducting their research this way for over 70 years. Moreover, byproducts and noise typically are identifiable only after the adaptations of which they are a byproduct or noise have been discovered and described (Tooby \& Cosmides, 1990).

Although modern evolutionary psychological theories are relatively new, all psychological theories are evolutionary in nature: "All psychological theories-be they cognitive, social, developmental, personality, or clinical-imply the existence of internal psychological mechanisms" (Buss, 1995, p. 2). If the internal psychological mechanisms implied in any psychological theory were not the product of the evolutionary process, then they would be, by default, unscientific theories.

\section{Psychological Mechanisms as Information-Processing Modules}

An evolved psychological mechanism is an information-processing module that was selected throughout a species's evolutionary history because it reliably produced behavior that solved a particular adaptive problem (Tooby \& Cosmides, 1992). Evolved psychological mechanisms are understood in terms of their specific input, decision rules, and output (Buss, 1995). Each psychological mechanism evolved to take in a narrow range of information-information specific to a specific adaptive problem. The information (or input) that the organism receives signals 
the adaptive problem that is being confronted. The input (either internal or external) is then transformed into output (i.e., behavior, physiological activity, or input relayed to another psychological mechanism) via a decision rule, an if-then procedure. An example illustrating the input, decision rules, and output of a psychological mechanism is appropriate here.

Fruit can be either ripe or unripe. Because ripe fruit is more nutritious (calorically dense) than immature fruit, humans have developed a preference for ripe fruit. The decision rule regarding the selection of fruit might go something like, "If the fruit tastes sweet, then eat it." If all fruit were maximally saturated with sugar all of the time, then that particular decision rule would not exist. The output associated with this mechanism might be to eat the ripe fruit or disregard the unripe fruit. This example illustrates the fact that psychological mechanisms develop and operate without any conscious awareness or formal learning, and we are blind to their underlying logic. (Do you enjoy calorically dense fruit because it provides nutrition needed to carry out activities related to survival and reproduction, or do you simply enjoy sweet fruit?)

Tooby and Cosmides (1992) have written that the causal link between evolution and behavior is made through psychological mechanisms. That is, the filter of natural selection operates on psychological mechanisms that produce behavior. Natural selection cannot operate on behavior directly but instead operates on the genes associated with the psychological mechanisms that produce the behavior. Williams (1966) spoke similarly: "The selection of genes is mediated by the phenotype [psychological mechanism], and in order to be favorably selected, a gene must produce phenotypic reproductive success [adaptive behavior]" (p. 25).

\section{Psychological Mechanisms and Domain Specificity}

The vast majority of psychological mechanisms are presumed to be domain-specific. That is, the mind is composed of content-dependent machinery (i.e., physiological and psychological mechanisms) that is presumed to have evolved to solve a specific adaptive problem. Psychological mechanisms can also be expressed as cognitive biases that cause people to more readily attend to or make sense of some pieces of information relative to others. This presumption of domain specificity or modularity contrasts with the traditional position that humans are endowed with a general set of learning or reasoning mechanisms that are 
applied to any problem regardless of specific content (e.g., Atkinson \& Wheeler, 2004). A system that is domain-general or content-independent, however, is a system that lacks a priori knowledge about specific situations or problem domains (Tooby \& Cosmides, 1992). Such a system, when faced with a choice in a chain of decisions, must select from all behavioral possibilities (e.g., wink, jump, remember father, smile, point finger, scream). This problem of choosing among an infinite range of possibilities when only a small subset are appropriate has been described by researchers in artificial intelligence, linguistics, and other disciplines (see Tooby \& Cosmides, 1992, for a review).

Not only are there theoretical arguments against a contentindependent system, myriad evidence for domain specificity comes from, among other areas, evolutionary psychological theory and research (e.g., Cosmides, 1989; Cosmides \& Tooby, 1994; Flaxman \& Sherman, 2000; Pinker \& Bloom, 1990), cognitive research (e.g., Hirschfeld \& Gelman, 1994), studies of animal learning (e.g., Carey \& Gelman, 1991; Garcia, Ervin, \& Koelling, 1966), and the clinical neurological literature (e.g., Gazzaniga \& Smylie, 1983; Ramachandran, 1995; Sergent, Ohta, \& MacDonald, 1992). Practitioners of evolutionary psychology concede that relatively domain-general mechanisms that function, for example, to integrate and relay information between domain-specific mechanisms may exist, but the vast majority of mechanisms are presumed to be domain-specific.

Some of the controversy surrounding the modularity of the mind seems to be rooted in the use of the term domain. Psychologists have often used the term to refer to particular domains of life, such as the mating domain, kinship domain, and parenting domain. Many have assumed subsequently that labeling a mechanism as domain-specific restricts the proposed mechanism to a particular domain, and if evidence can be garnered to show that the mechanism functions in more than one domain (e.g., the mating domain and the kinship domain), then it is taken as evidence for the domain generality of the proposed mechanism. This, however, is incorrect. A domain, when referring to a psychological mechanism, is a selection pressure, an adaptive problem (Cosmides $\&$ Tooby, 1987). Domain, then, is synonymous with problem. That is, a domain-specific mechanism refers to a problem-specific mechanisma mechanism that evolved to solve a specific adaptive problem. So, although evolutionary and cognitive psychologists use the term domainspecific, perhaps some confusion could be avoided if the more accurate term problem-specific were employed instead. Although some psycho- 
logical mechanisms cut across different domains of life (e.g., face recognition, working memory, processing speed), they still solve specific problems. Working memory, for example, solves the specific problem of holding information in the mind for a brief period of time. It has been suggested that evolutionary and cognitive psychologists might be better off avoiding these contentious labels and simply describing the proposed mechanism and its function (D. M. Buss, personal communication, January 2005).

\section{Evolutionary Time Lags and the Environment of Evolutionary Adaptedness}

Because evolution is an excruciatingly slow process, extant humans and their minds are designed for the earlier environments of which they are a product. Our minds were not designed to solve the day-today problems of our modern society but instead were designed to solve the day-to-day problems of our evolutionary past. Examples of evolutionary time lags abound: our difficulty in learning to fear modern threats, such as guns and cars, and our near effortless learning to fear more ancient threats, such as snakes and spiders (Öhman \& Mineka, 2001); children's ease in learning biologically primary mathematic abilities, such as counting, and their difficulty in learning biologically secondary mathematic abilities, such as arithmetic (Geary, 1995); women not conceding to intercourse indiscriminately, even though modern contraception can eliminate the reproductive costs associated with intercourse; and our preference for sugar and fat, which was once adaptive, owing to their scarcity, but has now become maladaptive. These few examples illustrate that our modern behavior is best understood when placed in the context of our environment of evolutionary adaptedness.

The environment of evolutionary adaptedness is not a place or a time in history but a statistical composite of the selection pressures (i.e., the enduring properties, components, and elements) of a species's ancestral past, and more specifically the adaptations that characterize that past (Tooby \& Cosmides, 1990). That is, each adaptation evolved as a result of a specific set of selection pressures. Each adaptation, in principle, has a unique environment of evolutionary adaptedness, but there likely would have been significant overlap in the environments of related adaptations. Tooby and Cosmides (1990) and other practitioners of evolutionary psychology, however, use "Pleistocene" to refer to the human 
environment of evolutionary adaptedness because this time period, approximately 1.81-0.01 million years ago, was appropriate for virtually all adaptations of Homo sapiens sapiens.

Although our evolutionary past is not available for direct observation, the discovery and description of adaptations allows us to make inferences about our evolutionary past, and the characterization of adaptations is arguably the single most reliable way of learning about the past (Tooby \& Cosmides, 1990). Some adaptations provide unequivocal information about our ancestral past. Our cache of psychological mechanisms associated with navigating the social world tells us that our ancestors were a social species (e.g., Cosmides, 1989; Cummins, 1998; Kurzban, Tooby, \& Cosmides, 2001; Pinker \& Bloom, 1990; Trivers, 1971). A multitude of psychological mechanisms associated with cuckoldry avoidance tell us that female infidelity was a recurrent feature of our evolutionary past (Buss, Larsen, Westen, \& Semmelroth, 1992; Buss \& Shackelford, 1997; Goetz et al., 2005; Platek, 2003; Shackelford et al., 2002).

Some adaptations, however, do not make clear (at least on first inspection) their link with our ancestral past. There exists, for example, a mechanism present in the middle ear of all humans that is able to reduce sound intensity by as much as $30 \mathrm{db}$ in $50 \mathrm{~ms}$. The attenuation reflex, as it is known, acts by contracting muscles that pull the stirrup away from the oval window of the cochlea, preventing strong vibrations from damaging the inner ear. The attenuation reflex meets the characteristics of an adaptation (e.g., economic, efficient, reliable), yet it is not obvious what selection pressures drove the evolution of this adaptation. That is, what specific noises did our ancestors recurrently hear that would create this noise-reducing mechanism? That the muscles appear to contract as we are about to speak suggests that our own loud voices might have been the impetus for this adaptation. Moreover, sound attenuation is greater at low frequencies than at high ones (and humans speak at low frequencies), also suggesting that ululating was a recurrent (enough) feature of our evolutionary past. Thus, from discovering and describing adaptations, we can tentatively characterize aspects of our evolutionary environment.

\section{Evolutionary Psychology Is Not Sociobiology}

Those less familiar with evolutionary psychology often construe the approach as "sociobiology reborn." Although sociobiology, ethology, 
behavioral ecology, and evolutionary psychology share with each other evolution as a guiding framework, the programs are conceptually distinct, for at least two reasons (Buss, 1995; Crawford, 2000). First, the focus on evolved psychological mechanisms and their information processing is a unique and defining feature of evolutionary psychology. The input, decision rules, and output of psychological mechanisms are central to the analysis. Second, evolutionary psychology does not measure individuals' reproductive success or fitness and views this endeavor as fruitless. Because many sociobiologists have advocated measuring an individual's reproductive success to understand the adaptive value of behavior, the pejorative label "baby counting" has been applied to sociobiology. Evolutionary psychology rejects the premise that measuring fitness in a recent or current environment provides any information about a particular behavior. The information needed to measure fitness correctly becomes known only generations later because there is no guarantee that selection pressures remain stable over time. Practitioners of evolutionary psychology hold that "humans are adaptation executers, not fitness maximizers" (Tooby \& Cosmides, 1990, p. 420). While many agree that evolutionary psychology is a separate field from other adaptationist programs, others hold that it is sociobiology in camouflage (e.g., Silverman, 2003).

\section{Evolutionary Psychology’s Future}

Although this modern approach to human behavior and cognition is relatively young, only about 25 years old, evolutionary psychology's impact is already permeating all areas of psychology and opening up lines of research missed entirely by previous psychologists.

Evolutionary psychology's merit and future are also demonstrated in the fact that the number of publications using an evolutionary psychological approach is growing exponentially (Durrant \& Ellis, 2003). Some have even suggested that in the foreseeable future, the psychological equivalent to Gray's Anatomy will be possible, describing a number of evolved psychological mechanisms, their information processing, and their neural substrates (Tooby \& Cosmides, 1992).

As new psychologists are impartially introduced to evolutionary psychology, as "traditional" (i.e., antievolutionary) psychologists retire, as evolutionary psychology's empirical harvest grows, as findings from genetics corroborate findings from evolutionary psychology (e.g., Cherkas et al., 2004), as the neural substrates underlying hypothesized 
psychological mechanisms are discovered (e.g., Platek, Keenan, \& Mohamed, 2006), and as cross-disciplinary frameworks of evidence are utilized (Schmitt \& Pilcher, 2004), evolutionary psychology is expected to emerge as the metatheory for psychological science.

\section{Conclusion}

In this chapter we introduced evolutionary theory and its modern impact on psychological science. We discussed how, with a focus on evolved psychological mechanisms and their information processing, evolutionary psychology has arisen as a compelling and fruitful approach to the study of human behavior and cognition.

Because the design of the mind owes its functional organization to a natural, evolutionary process, an evolutionarily psychological approach is a logical framework on which to base all psychological theories. Evolutionary psychological theories specify what problems our cognitive mechanisms were designed to solve, thereby providing important information about what their design features are likely to be. In other words, "Is it not reasonable to anticipate that our understanding of the human mind would be aided greatly by knowing the purpose for which it was designed?" (Williams, 1966, p. 16).

It is possible to do research in psychology with little or no knowledge of evolution. Most psychologists do. But without an evolutionary perspective, psychology becomes a disparate set of fields. Evolutionary explanations pervade all fields in psychology and provide a unifying metatheoretical framework within which all of psychological science can be organized.

\section{References}

Atkinson A. P., \& Wheeler, M. (2004). The grain of domains: The evolutionarypsychological case against domain-general cognition. Mind and Language, 19, 147-176.

Barkow, J. H., Cosmides, L., \& Tooby J. (1992). The adapted mind: Evolutionary psychology and the generation of culture. New York: Oxford University Press.

Buss, D. M. (1995). Evolutionary psychology: A new paradigm for psychological science. Psychological Inquiry, 6, 1-20.

Buss, D. M., Haselton, M. G., Shackelford, T. K., Bleske, A. L., \& Wakefield, J. C. (1998). Adaptations, exaptations, and spandrels. American Psychologist, 53, 533-548. 
Buss, D. M., Larsen, R., Westen, D., \& Semmelroth, J. (1992). Sex differences in jealousy: Evolution, physiology, and psychology. Psychological Science, 3, 251-255.

Buss, D. M., \& Shackelford, T. K. (1997). From vigilance to violence: Mate retention tactics in married couples. Journal of Personality and Social Psychology, 72, 346-361.

Carey, S., \& Gelman, R. (1991). The epigenesis of mind: Essays on biology and cognition. Hillsdale, NJ: Erlbaum.

Chagnon, N. A., \& Irons, W. (1979). Evolutionary biology and human social behavior: An anthropological perspective. North Scituate, MA: Duxbury Press.

Cherkas, L. F., Oelsner, E. C., Mak, Y. T., Valdes, A., \& Spector, T. (2004). Genetic influences on female infidelity and number of sexual partners in humans: A linkage and association study of the role of the vasopressin receptor gene (AVPR1A). Twin Research, 7, 649-658.

Cosmides, L. (1989). The logic of social exchange: Has natural selection shaped how humans reason? Studies with the Wason selection task. Cognition, 31, 187-276.

Cosmides, L., \& Tooby. J. (1987). From evolution to behavior: Evolutionary psychology as the missing link. In J. Dupre (Ed.), The latest on the best: Essays on evolution and optimality (pp. 277-306). Cambridge, MA: Bradford Books/MIT Press.

Cosmides, L., \& Tooby, J. (1994). Origins of domain specificity: The evolution of functional organization. In L. A. Hirschfeld and S. A. Gelman (Eds.), Mapping the mind: Domain specificity in cognition and culture (pp. 85-116). New York: Cambridge University Press.

Crawford, C. (2000). Evolutionary psychology: Counting babies or studying information processing mechanisms. Annals of the New York Academy of Sciences, 907, 21-38.

Cummins, D. D. (1998). Social norms and other minds: The evolutionary roots of higher cognition. In D. D. Cummins \& C. Allen (Eds.), The evolution of mind (pp. 30-50). New York: Oxford University Press.

Daly, M., \& Wilson, M. (1983). Sex, evolution, and behavior (2nd ed.). Boston: Willard Grant.

Darwin, C. (1859). On the origin of species. London: Murray.

Darwin, C. (1871). The descent of man and Selection in relation to sex. London: Murray.

Durrant, R., \& Ellis, B. J. (2003). Evolutionary psychology: Core assumptions and methodology. In M. Gallagher \& R. J. Nelson (Eds.), Comprehensive handbook of psychology, Vol. 3: Biological psychology (pp. 1-33). New York: John Wiley \& Sons.

Flaxman, S. M., \& Sherman, P. (2000). Morning sickness: A mechanism for protecting mother and embryo. The Quarterly Review of Biology, 75, 113-148.

Fisher, R. A. (1954). Retrospect of the criticisms of the theory of natural selection. In J. S. Huxley, A. C. Hardy, \& E. B. Ford (Eds.), Evolution as a process (pp. 84-98). London: Allen \& Unwin. 
Futuyma, D. J. (1986). Evolutionary biology (2nd ed.). Sunderland, MA: Sinauer Associates.

Garcia, J., Ervin, F. R., \& Koelling, R. A. (1966). Learning with prolonged delay of reinforcement. Psychonomic Science, 5, 121-122.

Gaulin S. J. C., \& McBurney, D. H. (2004). Evolutionary psychology (2nd ed.). Upper Saddle River, NJ: Pearson Education.

Gazzaniga, M. S., \& Smylie, C. S. (1983). Facial recognition and brain asymmetries: Clues to underlying mechanisms. Annals of Neurology, 13, $536-540$.

Geary, D. C. (1995). Reflections on evolution and culture in children's cognition: Implications for mathematical development and instruction. American Psychologist, 50, 24-37.

Goetz, A. T., Shackelford, T. K., Weekes-Shackelford, V. A., Euler, H. A., Hoier, S., Schmitt, D. P., \& LaMunyon, C. W. (2005). Mate retention, semen displacement, and human sperm competition: Tactics to prevent and correct female infidelity. Personality and Individual Differences, 38, 749-763.

Hamilton, W. D. (1964). The genetical evolution of social behaviour. Journal of Theoretical Biology, 7, 1-52.

Hamilton, W. D., \& Zuk, M. (1982). Heritable true fitness and bright birds: A role for parasites? Science, 218, 384-387.

Hirschfeld, L. A., \& Gelman, S. A. (1994). Mapping the mind: Domain specificity in cognition and culture. New York: Cambridge University Press.

Kettlewell, H. B. D. (1955). Selection experiments on industrial melanism in the Lepidoptera. Heredity, 9, 323-342.

Kettlewell, H. B. D. (1956). Further selection experiments on industrial melanism in the Lepidoptera. Heredity, 10, 287-301.

Kurzban, R., Tooby, J., \& Cosmides, L. (2001). Can race be erased? Coalitional computation and social categorization. Proceedings of the National Academy of Sciences, U.S.A., 98, 15387-15392.

Mayr, E. (1963). Animal species and their evolution. Cambridge, MA: Harvard University Press.

Mayr, E. (1982). The growth of biological thought: Diversity, evolution, and inheritance. Cambridge, MA: Harvard University Press.

Mayr, E., \& Provine, W. B. (1980). The evolutionary synthesis: Perspectives on the unification of biology. Cambridge, MA: Harvard University Press.

Öhman, A., \& Mineka, S. (2001). Fears, phobias, and preparedness: Toward an evolved module of fear and fear learning. Psychological Review, 108, 483-522.

Pinker, S., \& Bloom, P. (1990). Natural language and natural selection. Behavioral and Brain Sciences, 13, 707-727.

Platek, S. M., Keenan, J. P., \& Mohamed, F. B. (2005). Sex differences in the neural correlates of child facial resemblance: An event-related fMRI study. Neuroimage, 25, 1336-1344.

Platek, S. M. (2003). An evolutionary model of the effects of human paternal resemblance on paternal investment. Evolution and Cognition, 9, 189-197. 
Ramachandran, V. S. (1995). Anosognosia in parietal lobe syndrome. Consciousness and Cognition, 4, 22-51.

Schmitt, D. P., \& Pilcher, J. J. (2004). Evaluating evidence of psychological adaptation. Psychological Science, 15, 643-649.

Sergent, J., Ohta, S., \& MacDonald, B. (1992). Functional neuroanatomy of face and object processing. Brain, 115, 15-36.

Shackelford, T. K., LeBlanc, G. J., Weekes-Shackelford, V. A., Bleske-Rechek, A. L., Euler, H. A., \& Hoier, S. (2002). Psychological adaptation to human sperm competition. Evolution and Human Behavior, 23, 123-138.

Sherman, P. W. (1977). Nepotism and the evolution of alarm calls. Science, 197, 1246-1253.

Silverman, I. (2003). Confessions of a closet sociobiologist: Personal perspectives on the Darwinian movement in psychology. Evolutionary Psychology, 1, 1-9.

Symons, D. (1979). The evolution of human sexuality. New York: Oxford University Press.

Thornhill, R. (1997). The concept of an evolved adaptation. In G. R. Bock \& G. Cardew (Eds.), Characterizing human psychological adaptations (pp. 4-22). West Sussex, U.K.: John Wiley \& Sons.

Tooby, J., \& Cosmides, L. (1990). The past explains the present: Emotional adaptations and the structure of ancestral environments. Ethology and Sociobiology, 11, 375-424.

Tooby, J., \& Cosmides, L. (1992). The psychological foundations of culture. In J. H. Barkow, L. Cosmides, \& J. Tooby (Eds.), The adapted mind: Evolutionary psychology and the generation of culture (pp. 19-136). Oxford: Oxford University Press.

Trivers, R. L. (1971). The evolution of reciprocal altruism. Quarterly Review of Biology, 76, 35-57.

Williams, G. C. (1966). Adaptation and natural selection. Princeton, NJ: Princeton University Press. 